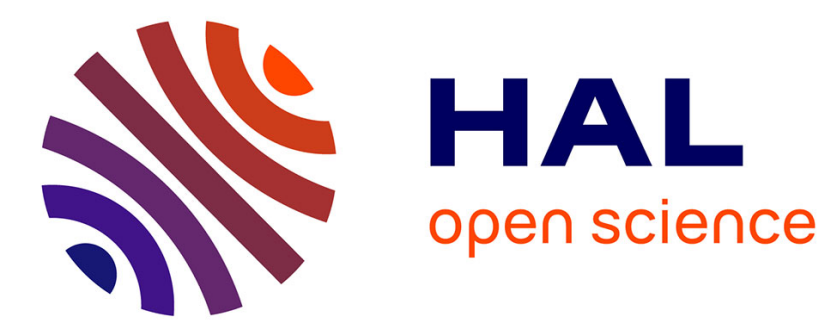

\title{
A descriptor Takagi-Sugeno approach to frequency weighted nonlinear model reduction
}

Benoît Marx

\section{To cite this version:}

Benoît Marx. A descriptor Takagi-Sugeno approach to frequency weighted nonlinear model reduction. IEEE International Conference on Fuzzy Systems, FuzzIEEE 2015, Aug 2015, Istanbul, Turkey. pp.17, 10.1109/FUZZ-IEEE.2015.7337985 . hal-01141266

\section{HAL Id: hal-01141266 https://hal.science/hal-01141266}

Submitted on 25 Nov 2021

HAL is a multi-disciplinary open access archive for the deposit and dissemination of scientific research documents, whether they are published or not. The documents may come from teaching and research institutions in France or abroad, or from public or private research centers.
L'archive ouverte pluridisciplinaire HAL, est destinée au dépôt et à la diffusion de documents scientifiques de niveau recherche, publiés ou non, émanant des établissements d'enseignement et de recherche français ou étrangers, des laboratoires publics ou privés. 


\title{
A descriptor Takagi-Sugeno approach to frequency weighted nonlinear model reduction
}

\author{
Benoît Marx
}

\begin{abstract}
In this note, the problem of frequency weighted nonlinear model reduction is addressed. It consists in approximating a given $n^{t h}$-order nonlinear system by a $k^{t h}$-order nonlinear system, where $k<n$. The goal is to compute the reduced order system minimizing the $\mathcal{L}_{2}$-gain from the input to the difference between the outputs of the original and the reduced systems. For this purpose the nonlinear system generating the approximation error is written under the descriptor Takagi-Sugeno formalism and is studied with the use of a multiple Lyapunov function, based on the descriptor approach. The obtained results are expressed in terms of Linear Matrix Inequalities (LMI) and the matrices defining the reduced order system are obtained as a result of LMI problem.
\end{abstract}

\section{INTRODUCTION}

Given an original system (of order $n$ ), the goal of model order reduction (MOR) is to find a system with a reduced order (of order $k$, with $k<n$ ) that suitably approximates the original system, according to a given norm criterion to be minimized. In general, a reduced order model is sought in order to be analyzed, simulated, or with the objective to design a controller (of reasonable order) for a complex process.

Different methods have arisen in the field of MOR, they can be roughly casted into three families: Krylov subspace method, truncated balanced realization (TBR) and $H_{\infty}$ approach. Krylov subspace based methods are based on the series expansion at a point of the matrix transfer of the system, see [4]. Theses methods became popular in high dimension circuit simulation where the same structure is repeated (e.g. a transmission line model identical RLC cells, see [9]) and thus can be projected in a vector base of limited dimension. The main drawback of this method is that the reduced model approximates well the original transfer mainly around a specified frequency. Krylov MOR are extended to the nonlinear case via linearization around some points [17], [9], [10]. Another group of MOR methods, appeared after [8], is based on Hankel norm approximation and TBR [23], [11] and is suitable for LTI systems. Roughly speaking, the minimization of the Hankel norm of the approximation error can be seen as the truncation of the least controllable and observable modes. The advantages of TBR methods are numerous: upper and lower bounds of the approximation error are known [8], [6], stability is preserved, frequency weighting functions can be introduced [3], [23], but the high computational cost is a limiting factor. The last set of MOR is based on $H_{\infty}$-optimization and is derived from

the author is with Université de Lorraine, CRAN, UMR 7039, 2 avenue de la forêt de Haye, 54516 Vandoeuvre-les-Nancy Cedex, France and with CNRS, CRAN, UMR 7039, France (e-mail: benoit.marx@univ-lorraine.fr). the $H_{\infty}$-control theory. Adopting this approach, the reduced order model is seen as a controller designed in order to minimize the $H_{\infty}$-gain of the transfer from the control input to the approximation error. In the case of LTI systems, [7] provides necessary and sufficient conditions for the existence of reduced systems. Since the obtained rank conditions are not linear, an iterative procedure is proposed. In [2] it is proved that the lower bound of the approximation error provided by $H_{\infty}$ method is the same than with TBR and a two-step MOR procedure is proposed. This procedure is improved in [5]. Due to the popular LMI formalism, $H_{\infty}$ model reduction have been extended to several classes of systems: singular systems [19], [21], switched LPV systems [20], switched systems with delay [18].

In this article, an extension of the MOR to nonlinear systems is proposed based on the $\mathcal{L}_{2}$-approach and on the Takagi-Sugeno (TS) formalism. Since [12], TS systems are extensively investigated due to their approximation properties: indeed, any nonlinear system can be exactly written (i.e. with a zero approximation error) as a TS system on a compact set of $\mathbb{R}^{n}$ [15]. This property motivates the choice of the TS approach. Beside the approximation property, one of the main advantages of the TS formalism is its closeness to linear formulation. In fact, a TS system is a time varying blending of LTI submodels. Consequently, numerous borrowings from the linear theory are possible [15]. As stated in the section II, the approximation error is quantified by the $\mathcal{L}_{2}$-gain from the input to the difference between the reduced an original system outputs, both in the TS form. As a result, computing the reduced order system is closely related to $\mathcal{L}_{2}$-norm bound properties. Some results on polyquadratic stability of TS systems [13], [14], are used to derive LMI conditions minimizing the $\mathcal{L}_{2}$-norm of a TS system. In section III, a constructive procedure is detailed in order to compute the matrices defining the reduced order system that minimizes the $\mathcal{L}_{2}$-gain of the error system. Frequency weighted MOR problem is treated in section IV. The results are illustrated in section $\mathrm{V}$, before concluding.

\section{PROBlem FORMULATION AND PRELIMINARIES}

Let a nonlinear system be described by the TS model

$$
\begin{aligned}
& \dot{x}(t)=\sum_{i=0}^{r} h_{i}(z(t))\left(A_{i} x(t)+B_{i} u(t)\right) \\
& y(t)=\sum_{i=1}^{r} h_{i}(z(t))\left(C_{i} x(t)+D_{i} u(t)\right)
\end{aligned}
$$


where $x(t) \in \mathbb{R}^{n}, u(t) \in \mathbb{R}^{p}, y(t) \in \mathbb{R}^{m}$ and $z(t) \in \mathbb{R}^{q}$ are respectively the state vector, the control input, the measured output and the decision variable. Each weighting function $h_{i}($.$) quantifies the relative importance of the i^{\text {th }}$ submodel $\left(A_{i}, B_{i}, C_{i}, D_{i}\right)$ in the global nonlinear system (1). These functions satisfy the convex sum properties

$$
\sum_{i=1}^{r} h_{i}(z(t))=1 \quad \text { and } \quad 0 \leq h_{i}(z(t)) \leq 1, \quad \forall t \geq 0
$$

Notations. The notation $*$ is used for the blocks induced by symmetry, for any square matrix $M, \mathbb{S}(M)$ is defined by $\mathbb{S}(M)=M+M^{T}, I_{n}$ is the $n \times n$ identity matrix, $0_{n}$ (resp. $0_{n \times m}$ ) is the $n \times n$ (resp. $n \times m$ ) null matrix. The matrix $M=\operatorname{diag}\left(M_{1}, M_{2}, \ldots, M_{q}\right)$ is a block diagonal matrix with the blocks $M_{1}, M_{2}, \ldots, M_{q}$ on its diagonal entries. For any sets of matrices $X_{i}(i=1, \ldots, r)$ and $Y_{i j}(i=1, \ldots, r$ and $j=1, \ldots, r)$, the polytopic matrices $X_{h}$ and $X_{h h}$ are respectively defined by

$$
X_{h}=\sum_{i=1}^{r} h_{i}(z(t)) X_{i} \quad Y_{h h}=\sum_{i=1}^{r} \sum_{j=1}^{r} h_{i}(z(t)) h_{j}(z(t)) Y_{i j}
$$

In the remaining of the paper, the activating functions are denoted $h_{i}(t)$ instead of $h_{i}(z(t))$. Similarly to [13], the two following assumptions are made in the remaining of this note.

(A1) the decision variable $z(t)$ is real time accessible (e.g. known exogenous signal, input signal).

(A2) the weighting functions satisfy $\left|\dot{h}_{i}(z(t))\right| \leq \Phi_{i}, \forall t>0$ and $i \in\{1, \ldots, r-1\}$.

The MOR problem can be formulated as finding the reduced order TS system, sharing the same input and decision variable that (1) such that the output of the reduced order system, denoted $y^{r}(t)$, is as close as possible to $y(t)$, the output of the original system. The numbers of submodels in both the reduced and the original models are equal to $r$. In fact, the complexity reduction comes from the choice of a vector state of lower dimension than the one of (1). The reduced system is defined by

$$
\begin{aligned}
& \dot{x}^{r}(t)=A_{h}^{r} x^{r}(t)+B_{h}^{r} u(t) \\
& y^{r}(t)=C_{h}^{r} x^{r}(t)+D_{h}^{r} u(t)
\end{aligned}
$$

with $x^{r}(t) \in \mathbb{R}^{k}$ with $k<n$ and $y^{r}(t) \in \mathbb{R}^{m}$. In order to quantify the distance between the two model outputs, the approximation error system is defined by the difference between the outputs of (1) and (4): $e(t)=y(t)-y^{r}(t)$. It is readily given by

$$
\begin{aligned}
\dot{\bar{x}}(t) & =\bar{A}_{h} \bar{x}(t)+\bar{B}_{h} u(t) \\
e(t) & =\bar{C}_{h} \bar{x}(t)+\bar{D}_{h} u(t)
\end{aligned}
$$

where $\bar{x}^{T}(t)=\left[x^{T}(t) x^{r T}(t)\right], \bar{A}_{i}=\operatorname{diag}\left(A_{i}, A_{i}^{r}\right), \bar{B}_{i}^{T}=$

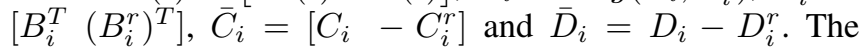
approximation error system can be written as a descriptor system

$$
\begin{aligned}
E \dot{x}_{a}(t) & =A_{h}^{*} x_{a}(t)+B_{h}^{*} u(t) \\
e(t) & =C_{h}^{*} x_{a}(t)+D_{h}^{*} u(t)
\end{aligned}
$$

where $x_{a}^{T}(t)=\left[\bar{x}^{T}(t) \bar{x}^{T}(t)\right], E=\operatorname{diag}\left(I_{n+k}, 0_{n+k}\right)$, $C_{i}^{*}=\left[\bar{C}_{i} 0_{m \times(n+k)}\right], D_{i}^{*}=\bar{D}_{i}$ and

$$
A_{i}^{*}=\left(\begin{array}{cc}
\bar{A}_{i} & 0_{n+k} \\
I_{n+k} & -I_{n+k}
\end{array}\right) B_{i}^{*}=\left(\begin{array}{c}
\bar{B}_{i} \\
0_{(n+k) \times p}
\end{array}\right)
$$

As discussed in [14], the interest of the descriptor approach is to introduce some degree of freedom (DOF) in the optimization problem. It must be pointed out, that no impulsive behavior is introduced since the restriction of $\sum_{i=1}^{r} h_{i}(z(t)) A_{i}$ in the right kernel of $E$ is invertible. The approximation error is given by the $\mathcal{L}_{2}$-gain of (6) from $u(t)$ to $e(t)=y(t)-y^{r}(t)$, that is to say the upper bound of the ratio of the energy of these signals. It is well known (e.g. [1]) that the $\mathcal{L}_{2}$-gain from $u$ to $e$ is upper bounded by $\gamma>0$ if there exists a Lyapunov function $V\left(x_{a}\right)$ verifying

$$
\dot{V}\left(x_{a}(t)\right)+e^{T}(t) e(t)-\gamma^{2} u^{T}(t) u(t)<0, \quad \forall t>0
$$

The MOR problem reduces to find the matrices $A_{i}^{r}, B_{i}^{r}, C_{i}^{r}$ and $D_{i}^{r}$ (for $i \in\{1, \ldots, r\}$ ) minimizing the $\mathcal{L}_{2}$-gain of the approximation error system (5) or (6). This minimization is based on the Lyapunov function defined by

$$
V\left(x_{a}(t)\right)=x_{a}^{T}(t) E^{T}\left(X_{h}\right)^{-1} x_{a}(t)
$$

with $X_{i}^{T} E^{T}=E X_{i} \geq 0$ for $i=1, \ldots, r$, proposed in [14].

Before detailing the main results, useful lemmas, taken from [16] and [24] respectively, are recalled.

Lemma 1: For any $h_{i}$ satisfying (2), the inequality $X_{h h}<$ 0 holds if the following inequalities are satisfied

$$
\begin{aligned}
X_{i i} & <0, \text { for } 1 \leq i \leq r \\
\frac{1}{r-1} X_{i i}+\frac{1}{2}\left(X_{i j}+X_{j i}\right) & <0, \text { for } 1 \leq i \neq j \leq r
\end{aligned}
$$

Lemma 2: For any scalar $\tau>0$ and matrices $X, \Sigma(t), Y$ of appropriate dimensions with $\Sigma^{T}(t) \Sigma(t) \leq I$, it follows

$$
X^{T} \Sigma^{T}(t) Y+Y^{T} \Sigma(t) X \leq \tau X^{T} X+\tau^{-1} Y^{T} Y
$$

\section{DESIGN OF THE REDUCED ORDER SYSTEM}

Adopting the $\mathcal{L}_{2}$-approach, the MOR problem is reduced to an $\mathcal{L}_{2}$-controller design where the reduced order system is considered as the controller, designed in order to control the approximation error. The result proposed here is based on the relaxed stability conditions given in [14] and also on the relaxation introduced by [16]. In this section, the MOR problem for TS systems is expressed as a problem of minimization under LMI constraints. Once the minimization problem is solved, some of the obtained LMI variables are used to compute the gains of the reduced order system. This method does not provide any apriori information on the $\mathcal{L}_{2}$ gain of the approximation error (no lower or upper bound like in TBR methods for linear models), but the gain is obtained simultaneously with the matrices defining the reduced order model as a result of the optimization process. With this quantitative indicator, it can be appreciated whether the order of the reduced system is sufficiently large to have a precise approximation of the original system. 
Theorem 1: There exists a reduced system (4) of order $k<n$ approximating the system (1) (i.e. minimizing the $\mathcal{L}_{2}$ gain from $u(t)$ to $e(t)$ in (5)), if there exist matrices $X_{i}^{11}=$ $X_{i}^{11 T}, X_{i}^{31}$ and $X_{i}^{33} \in \mathbb{R}^{n \times n}$, matrices $X^{12}, X_{i}^{32}$ and $X_{i}^{34} \in$ $\mathbb{R}^{n \times k}$, matrices $X^{22}=X^{22 T}, X_{i}^{42}, X_{i}^{44}$ and $A_{2 i}^{r} \in \mathbb{R}^{k \times k}$, matrices $X_{i}^{41}, X_{i}^{43}$ and $A_{1 i}^{r} \in \mathbb{R}^{k \times n}$, matrices $C_{1 i}^{r} \in \mathbb{R}^{m \times n}$ and $C_{2 i}^{r} \in \mathbb{R}^{m \times k}$, matrices $B_{i}^{r} \in \mathbb{R}^{k \times p}$ and matrices $D_{i}^{r} \in$ $\mathbb{R}^{m \times p}$, minimizing $\bar{\gamma}$ under the LMI constraints (13-16).

$$
\begin{aligned}
{\left[\begin{array}{cc}
X_{i}^{11} & X^{12} \\
X^{12 T} & X^{22}
\end{array}\right]>0,1 \leq i \leq r } \\
X_{i}^{11}-X_{r}^{11} \leq 0,1 \leq i \leq r-1 \\
\Theta_{i i}<0,1 \leq i \leq r \\
\frac{1}{r-1} \Theta_{i i}+\frac{1}{2}\left(\Theta_{i j}+\Theta_{j i}\right)<0,1 \leq i \neq j \leq r
\end{aligned}
$$

with

$$
\Theta_{i j}=\left[\begin{array}{ccc}
\Theta_{i j}^{11} & * & * \\
\vdots & \ddots & * \\
\Theta_{i j}^{61} & \ldots & \Theta_{i j}^{66}
\end{array}\right]
$$

where the $\Theta_{i j}^{k \ell}$ are given by:

$$
\begin{array}{rlrl}
\Theta_{i j}^{11} & =\mathbb{S}\left(A_{i} X_{j}^{11}\right)-\sum_{k=1}^{r-1} & \Phi_{k}\left(X_{k}^{11}-X_{r}^{11}\right) \\
\Theta_{i j}^{21} & =A_{1 i}^{r}+\left(A_{i} X^{12}\right)^{T} & \Theta_{i j}^{22} & =\mathbb{S}\left(A_{2 i}^{r}\right) \\
\Theta_{i j}^{31} & =X_{j}^{11}-X_{j}^{31} & \Theta_{i j}^{32} & =X^{12}-X_{j}^{32} \\
\Theta_{i j}^{33} & =-\mathbb{S}\left(X_{j}^{33}\right) & \Theta_{i j}^{41} & =X^{12 T}-X_{j}^{41} \\
\Theta_{i j}^{42} & =X^{22}-X_{j}^{42} & \Theta_{i j}^{43} & =-X_{j}^{43}-X_{j}^{34 T} \\
\Theta_{i j}^{44} & =-\mathbb{S}\left(X_{j}^{44}\right) & \Theta_{i j}^{51} & =B_{i}^{T} \\
\Theta_{i j}^{52} & =B_{i}^{r T} & \Theta_{i j}^{53} & =0 \\
\Theta_{i j}^{54} & =0 & \Theta_{i j}^{55} & =-\bar{\gamma} I_{p} \\
\Theta_{i j}^{61} & =C_{i} X_{j}^{11}-C_{1 i}^{r} & \Theta_{i j}^{62} & =C_{i} X^{12}-C_{2 i}^{r} \\
\Theta_{i j}^{63} & =0 & \Theta_{i j}^{64} & =0 \\
\Theta_{i j}^{65} & =D_{i}-D_{i}^{r} & \Theta_{i j}^{66} & =-I_{m}
\end{array}
$$

The $\mathcal{L}_{2}$-gain from $u(t)$ to $e(t)$ is given by $\gamma=\sqrt{\bar{\gamma}}$ and the matrices $A_{i}^{r}$ and $C_{i}^{r}$ are respectively obtained by

$$
\begin{aligned}
& A_{i}^{r}=\left(A_{1 i}^{r} X^{12}+A_{2 i}^{r} X^{22}\right)\left(X^{12 T} X^{12}+X^{22} X^{22}\right)^{-1} \\
& C_{i}^{r}=\left(C_{1 i}^{r} X^{12}+C_{2 i}^{r} X^{22}\right)\left(X^{12 T} X^{12}+X^{22} X^{22}\right)^{-1}
\end{aligned}
$$

Proof: The considered Lyapunov function is (9) with

$$
X_{i}=\left[\begin{array}{cccc}
X_{i}^{11} & X^{12} & 0 & 0 \\
X^{12 T} & X^{22} & 0 & 0 \\
X_{i}^{31} & X_{i}^{32} & X_{i}^{33} & X_{i}^{34} \\
X_{i}^{41} & X_{i}^{42} & X_{i}^{43} & X_{i}^{44}
\end{array}\right]
$$

From the two first rows and columns of (13) (resp. the third and fourth rows and columns of (15)) it follows that the submatrices obtained by selecting the two first rows and columns (resp. the two last rows and columns) of $X_{i}$ are positive definite. Due to the block triangular structure of $X_{i}$, it follows that the matrices $X_{i}$ are nonsingular. The time derivative of (9) is

$$
\dot{V}(t)=x_{a}^{T}(t) E^{T} \frac{d}{d t}\left(X_{h}\right)^{-1} x_{a}(t)+\mathbb{S}\left(\dot{x}_{a}^{T}(t) E^{T} X_{h}^{-1} x_{a}(t)\right)
$$

From (20) and (7), it follows $E X_{i}=X_{i}^{T} E^{T}$. With the positivity of the functions $h_{i}$, (13) ensures

$$
E^{T}\left(X_{h}\right)^{-1}=\left(X_{h}\right)^{-T} E \geq 0
$$

Moreover, derivating (2) it follows that $\sum_{i=1}^{r} \dot{h}_{i}(t)=0$, which results in $\dot{h}_{r}(t)=-\sum_{i=1}^{r-1} \dot{h}_{i}(t)$, the second term in (21) can be developed as

$$
\begin{aligned}
\frac{d}{d t}\left(X_{h}\right)^{-1} & =-\left(X_{h}\right)^{-1}\left(\sum_{i=1}^{r} \frac{d}{d t} h_{i}(t) X_{i}\right)\left(X_{h}\right)^{-1} \\
& =-\left(X_{h}\right)^{-1}\left(\sum_{i=1}^{r-1} \dot{h}_{i}(t)\left(X_{i}-X_{r}\right)\right)\left(X_{h}\right)^{-1}
\end{aligned}
$$

With (6), (22) and (23), the derivative of (9) becomes

$$
\begin{aligned}
\dot{V}(t)= & -x_{a}^{T}(t) X_{h}^{-T}\left(\sum_{i=1}^{r-1} \dot{h}_{i}(t) E\left(X_{i}-X_{r}\right)\right) X_{h}^{-1} x_{a}(t) \\
& +\mathbb{S}\left(\left(A_{h}^{*} x_{a}(t)+B_{h}^{*} u(t)\right)^{T}\left(X_{h}\right)^{-1} x_{a}(t)\right)
\end{aligned}
$$

Let denote $\Gamma(t)=\dot{V}\left(x_{a}(t)\right)+e^{T}(t) e(t)-\gamma^{2} u^{T}(t) u(t)$, the left hand side of (8) is given by $\Gamma(t)=\xi^{T}(t) \mathcal{M}(t) \xi(t)$ where $\xi^{T}(t)=\left[x_{a}^{T}(t) u^{T}(t)\right]$ and $\mathcal{M}(t)$ is defined by

$$
\mathcal{M}(t)=\left[\begin{array}{ll}
\mathcal{M}_{1}(t) & \mathcal{M}_{2}(t) \\
\mathcal{M}_{2}^{T}(t) & \mathcal{M}_{3}(t)
\end{array}\right]
$$

with

$$
\begin{aligned}
\mathcal{M}_{1}(t)= & -\left(X_{h}\right)^{-T}\left(\sum_{i=1}^{r-1} \dot{h}_{i}(z(t)) E\left(X_{i}-X_{r}\right)\right)\left(X_{h}\right)^{-1} \\
& +\mathbb{S}\left(\left(X_{h}\right)^{-T} A_{h}^{*}\right)+\left(C_{h}^{*}\right)^{T} C_{h}^{*} \\
\mathcal{M}_{2}(t)= & \left(X_{h}\right)^{-T} B_{h}^{*}+\left(C_{h}^{*}\right)^{T} D_{h}^{*} \\
\mathcal{M}_{3}(t)= & \left(D_{h}^{*}\right)^{T} D_{h}^{*}-\gamma^{2} I_{p}
\end{aligned}
$$

The inequality (8) is satisfied if and only if $\mathcal{M}(t)<0$. Applying a Schur complement, $\mathcal{M}(t)<0$ is equivalent to

$$
\left[\begin{array}{ccc}
\mathcal{M}_{1 a}(t) & * & * \\
\left(B_{h}^{*}\right)^{T}\left(X_{h}\right)^{-1} & -\gamma^{2} I_{p} & * \\
C_{h}^{*} & D_{h}^{*} & -I_{m}
\end{array}\right]<0
$$

with $\mathcal{M}_{1 a}(t)=-X_{h}^{-T}\left(\sum_{k=1}^{r-1} \dot{h}_{k}(t) E\left(X_{k}-X_{r}\right)\right) X_{h}^{-1}+$ $\mathbb{S}\left(X_{h}^{-T} A_{h}^{*}\right)$. Pre- and post-multiplying (26) by $T=$ $\operatorname{diag}\left(\left(X_{h}\right)^{T}, I_{p}, I_{n+k}\right)$ and $T^{T}$ respectively, it follows

$$
\left[\begin{array}{ccc}
\mathcal{M}_{1 b}(t) & * & * \\
\left(B_{h}^{*}\right)^{T} & -\gamma^{2} I_{p} & * \\
C_{h}^{*} X_{h} & D_{h}^{*} & -I_{m}
\end{array}\right]<0
$$

with

$$
\mathcal{M}_{1 b}(t)=-\left(\sum_{k=1}^{r-1} \dot{h}_{k}(z(t)) E\left(X_{k}-X_{r}\right)\right)+\mathbb{S}\left(A_{h}^{*} X_{h}\right)
$$


The inequality (14) is equivalent to $E\left(X_{k}-X_{r}\right) \leq 0$. With $\left|\dot{h}_{k}(z(t))\right| \leq \Phi_{k}$, it follows

$$
-\sum_{k=1}^{r-1} \dot{h}_{k}(t) E\left(X_{k}-X_{r}\right) \leq-\sum_{k=1}^{r-1} \Phi_{k} E\left(X_{k}-X_{r}\right)
$$

From (27) and (29), it follows that the $\mathcal{L}_{2}$-gain of the approximation error is bounded by $\gamma$ (i.e. (8) is satisfied) if $\mathcal{M}_{h h}<0$ holds with

$$
\mathcal{M}_{i j}=\left[\begin{array}{ccc}
\mathcal{M}_{i j}^{11} & * & * \\
B_{i}^{* T} & -\gamma^{2} I_{p} & * \\
C_{i}^{*} X_{j} & D_{i}^{*} & -I_{m}
\end{array}\right]
$$

where $\mathcal{M}_{i j}^{11}=-\sum_{k=1}^{r-1} \Phi_{k} E\left(X_{k}-X_{r}\right)+\mathbb{S}\left(A_{i}^{*} X_{j}\right)$. Using the lemma 1 , sufficient conditions are given by

$$
\begin{aligned}
\mathcal{M}_{i i} & <0,1 \leq i \leq r \\
\frac{1}{r-1} \mathcal{M}_{i i}+\frac{1}{2}\left(\mathcal{M}_{i j}+\mathcal{M}_{j i}\right) & <0,1 \leq i \neq j \leq r
\end{aligned}
$$

These inequalities are not linear in the sought matrices $X_{i}$, $A_{i}^{r}$ and $C_{i}^{r}$. In order to obtain LMI, the following variable changes are defined by $\bar{\gamma}=\gamma^{2}$ and

$$
\begin{aligned}
& {\left[\begin{array}{ll}
A_{1 i}^{r} & A_{2 i}^{r}
\end{array}\right]=A_{i}^{r}\left[\begin{array}{ll}
X^{12 T} & X^{22}
\end{array}\right]} \\
& {\left[\begin{array}{ll}
C_{1 i}^{r} & C_{2 i}^{r}
\end{array}\right]=C_{i}^{r}\left[\begin{array}{ll}
X^{12 T} & X^{22}
\end{array}\right]}
\end{aligned}
$$

With the definition of $X_{i}$ given by (20) and the variable changes defined by (32-33) it follows

$$
\begin{aligned}
A_{i}^{*} X_{j} & =\left[\begin{array}{cccc}
A_{i} X_{j}^{11} & A_{i} X^{12} & 0 & 0 \\
A_{1 i}^{r} & A_{2 i}^{r} & 0 & 0 \\
X_{j}^{11}-X_{j}^{31} & X^{12}-X_{j}^{32} & -X_{j}^{33} & -X_{j}^{34} \\
X^{12 T}-X_{j}^{41} & X^{22}-X_{j}^{42} & -X_{j}^{43} & -X_{j}^{44}
\end{array}\right] \\
C_{i}^{*} X_{j} & =\left[\begin{array}{llll}
C_{i} X_{j}^{11}-C_{1 i}^{r} & C_{i} X^{12}-C_{2 i}^{r} & 0 & 0
\end{array}\right]
\end{aligned}
$$

Thus, with (34), the inequality (31) becomes (15). Since the matrices $X_{i}$ are non singular, the matrix $\left[X^{12 T} X^{22}\right]$ is full row rank and thus $A_{i}^{r}$ and $C_{i}^{r}$ can be obtained by right pseudo inversion of $\left[X^{12 T} X^{22}\right]$ in (32) and (33) and are given by (18) and (19), achieving the proof.

\section{MOR WITH FREQUENCY WEIGHTING}

Frequency weighting functions can be introduced in the MOR procedure if this procedure appears to be too conservative to be tractable. Such functions may be a mean to relax the specifications in some frequency range(s) of less interest since the requirement on the approximation accuracy is not the same at the whole frequency range [22]. In order to do so, it suffices to chose a weighting function with a gain significantly less than 1 in the frequency range(s) of less interest. In this case, the criterion to be minimized is the $\mathcal{L}_{2}$-gain from the input $u(t)$ to the filtered approximation error $e^{f}(t)$, where $e^{f}(t)$ is the output of the state space system $\left(A^{f}, B^{f}, C^{f}, D^{f}\right)$ of order $n_{f}$, fed with $e(t)$ defined in (5b). The system matrices $A^{f}, B^{f}, C^{f}$ and $D^{f}$ are chosen by the user in order to put an emphasis on prescribed frequency ranges while relaxing the $\mathcal{L}_{2}$-gain constraints in the remaining. In this case, the approximation error becomes

$$
\begin{aligned}
\dot{\bar{x}}^{f}(t) & =\bar{A}_{h}^{f} \bar{x}^{f}(t)+\bar{B}_{h}^{f} u(t) \\
e^{f}(t) & =\bar{C}_{h}^{f} \bar{x}^{f}(t)+\bar{D}_{h}^{f} u(t)
\end{aligned}
$$

with $\quad \bar{x}(t)=\left[\begin{array}{lll}x^{f T}(t) & x^{T}(t) & x^{r T}(t)\end{array}\right], \quad \bar{C}_{i}^{f}=$ $\left[\begin{array}{lll}C^{f} & D^{f} C_{i} & -D^{f} C_{i}^{r}\end{array}\right], \bar{D}_{i}^{f}=D^{f}\left(D_{i}-D_{i}^{r}\right)$ and

$$
\bar{A}_{i}^{f}=\left[\begin{array}{ccc}
A^{f} & B^{f} C_{i} & -B^{f} C_{i}^{r} \\
0 & A_{i} & 0 \\
0 & 0 & A_{i}^{r}
\end{array}\right] \quad \bar{B}_{i}^{f}=\left[\begin{array}{c}
B^{f}\left(D_{i}-D_{i}^{r}\right) \\
B_{i} \\
B_{i}^{r}
\end{array}\right]
$$

The following theorem details the computation of the reduced order system $\left(A_{i}^{r}, B_{i}^{r}, C_{i}^{r}, D_{i}^{r}\right)$ of order $k$ for given frequency weighting system $W^{f}(s)$.

Theorem 2: There exists a reduced system (4) of order $k<n$ optimally approximating the system (35) (i.e. minimizing the $\mathcal{L}_{2}$-gain from $u(t)$ to $e^{f}(t)$ in (35)), if there exist matrices $X_{i}^{11}=X_{i}^{11 T}, X_{i}^{41}$ and $X_{i}^{44} \in \mathbb{R}^{n_{f} \times n_{f}}$, matrices $X_{i}^{12}, X_{i}^{42}$ and $X_{i}^{45} \in \mathbb{R}^{n_{f} \times n}$, matrices $X^{13}$, $X_{i}^{43}$ and $X_{i}^{46} \in \mathbb{R}^{n_{f} \times k}$, matrices $X_{i}^{22}=X_{i}^{22 T}, X_{i}^{52}$ and $X_{i}^{55} \in \mathbb{R}^{n \times n}$, matrices $X^{23}, X_{i}^{53}$ and $X_{i}^{56} \in \mathbb{R}^{n \times k}$, matrices $X^{33}=X^{33 T}, X_{i}^{63}, X_{i}^{66}$ and $A_{3 i}^{r} \in \mathbb{R}^{k \times k}$, matrices $X_{i}^{51}$ and $X_{i}^{54} \in \mathbb{R}^{n \times n_{f}}$, matrices $X_{i}^{61}, X_{i}^{64}$ and $A_{1 i}^{r} \in \mathbb{R}^{k \times n_{f}}$, matrices $X_{i}^{62}, X_{i}^{65}$ and $A_{2 i}^{r} \in \mathbb{R}^{k \times n}$, matrices $C_{1 i}^{r} \in$ $\mathbb{R}^{m \times n_{f}}, C_{2 i}^{r} \in \mathbb{R}^{m \times n}, C_{3 i}^{r} \in \mathbb{R}^{m \times k}$ matrices $B_{i}^{r} \in \mathbb{R}^{k \times p}$ and matrices $D_{i}^{r} \in \mathbb{R}^{m \times p}$, minimizing $\bar{\gamma}$ under the LMI constraints (37-40).

$$
\begin{gathered}
{\left[\begin{array}{ccc}
X_{i}^{11} & X_{i}^{12} & X^{13} \\
X_{i}^{12 T} & X_{i}^{22} & X^{23} \\
X^{13 T} & X^{23 T} & X^{33}
\end{array}\right]>0,1 \leq i \leq r} \\
{\left[\begin{array}{cc}
X_{i}^{11} & X_{i}^{12} \\
X_{i}^{12 T} & X_{i}^{22}
\end{array}\right]-\left[\begin{array}{cc}
X_{r}^{11} & X_{r}^{12} \\
X_{r}^{12 T} & X_{r}^{22}
\end{array}\right] \leq 0,1 \leq i \leq r-1} \\
\Theta_{i i}<0,1 \leq i \leq r \\
\frac{1}{r-1} \Theta_{i i}+\frac{1}{2}\left(\Theta_{i j}+\Theta_{j i}\right)<0,1 \leq i \neq j \leq r
\end{gathered}
$$

where $\Theta_{i j}$ is defined by

$$
\Theta_{i j}=\left[\begin{array}{ccc}
\Theta_{i j}^{11} & * & * \\
\vdots & \ddots & * \\
\Theta_{i j}^{81} & \ldots & \Theta_{i j}^{88}
\end{array}\right]
$$

with

$$
\begin{aligned}
\Theta_{i j}^{11}= & \mathbb{S}\left(A^{f} X_{j}^{11}+B^{f} C_{i} X_{j}^{12 T}-B^{f} C_{1 i}^{r}\right)-\sum_{k=1}^{r-1} \Phi_{k}\left(X_{k}^{11}-X_{r}^{11}\right) \\
\Theta_{i j}^{21}= & A_{i} X_{j}^{12 T}+\left(A^{f} X_{j}^{12}+B^{f} C_{i} X_{j}^{22}-B^{f} C_{2 i}^{r}\right)^{T} \\
& -\sum_{k=1}^{r-1} \Phi_{k}\left(X_{k}^{12}-X_{r}^{12}\right)^{T} \\
\Theta_{i j}^{22}= & \mathbb{S}\left(A_{i} X_{j}^{22}\right)-\sum_{k=1}^{r-1} \Phi_{k}\left(X_{k}^{22}-X_{r}^{22}\right) \\
\Theta_{i j}^{31}= & A_{1 i}^{r}+\left(A^{f} X^{13}+B^{f} C_{i} X^{23}-B^{f} C_{3 i}^{r}\right)^{T}
\end{aligned}
$$




$$
\begin{aligned}
& \Theta_{i j}^{32}=A_{2 i}^{r}+\left(A_{i} X^{23}\right)^{T} \\
& \Theta_{i j}^{33}=\mathbb{S}\left(A_{3 i}^{r}\right) \\
& \Theta_{i j}^{41}=X_{j}^{11}-X_{j}^{41} \\
& \Theta_{i j}^{43}=X^{13}-X_{j}^{43} \\
& \Theta_{i j}^{51}=X_{j}^{12 T}-X_{j}^{51} \\
& \Theta_{i j}^{53}=X^{23}-X_{j}^{53} \\
& \Theta_{i j}^{55}=-\mathbb{S}\left(X_{j}^{55}\right) \\
& \Theta_{i j}^{62}=X^{23 T}-X_{j}^{62} \\
& \Theta_{i j}^{64}=-X_{j}^{64}-X_{j}^{46 T} \\
& \Theta_{i j}^{66}=-\mathbb{S}\left(X_{j}^{66}\right) \\
& \Theta_{i j}^{72}=B_{i}^{T} \\
& \Theta_{i j}^{74}=0 \\
& \Theta_{i j}^{76}=0 \\
& \Theta_{i j}^{81}=C^{f} X_{j}^{11}+D^{f} C_{i} X_{j}^{12 T}- \\
& \Theta_{i j}^{82}=C^{f} X_{j}^{12}+D^{f} C_{i} X_{j}^{22}-\quad D^{f} C_{2 i}^{r} \\
& \Theta_{i j}^{83}=C^{f} X^{13}+D^{f} C_{i} X^{23}-\quad D^{f} C_{3 i}^{r} \\
& \Theta_{i j}^{84}=0 \\
& \Theta_{i j}^{85}=0 \\
& \Theta_{i j}^{86}=0 \\
& \Theta_{i j}^{88}=-I_{m}
\end{aligned}
$$

The $\mathcal{L}_{2}$-gain from $u(t)$ to $e^{f}(t)$ is given by $\gamma=\sqrt{\bar{\gamma}}$ and the matrices $A_{i}^{r}$ and $C_{i}^{r}$ are respectively obtained by

$$
\begin{aligned}
& A_{i}^{r}=\left(\sum_{k=1}^{3} A_{k i}^{r} X^{k 3}\right)\left(\sum_{k=1}^{3} X^{k 3 T} X^{k 3}\right)^{-1} \\
& C_{i}^{r}=\left(\sum_{k=1}^{3} C_{k i}^{r} X^{k 3}\right)\left(\sum_{k=1}^{3} X^{k 3 T} X^{k 3}\right)^{-1}
\end{aligned}
$$

Proof: Following the proof of theorem 1, the system (35) is written as the descriptor system (6) with $x_{a}^{T}(t)=\left[\begin{array}{ll}\bar{x}^{T}(t) & \bar{x}^{T}(t)\end{array}\right], C_{i}^{*}=\left[\begin{array}{ll}\bar{C}_{i}^{f} & 0\end{array}\right], D_{i}^{*}=\bar{D}_{i}^{f}, E=$ $\operatorname{diag}\left(I_{n_{f}+n+k}, 0_{n_{f}+n+k}\right)$ and

$$
\begin{aligned}
& A_{i}^{*}=\left[\begin{array}{cc}
\bar{A}_{i}^{f} & 0_{n_{f}+n+k} \\
I_{n_{f}+n+k} & -I_{n_{f}+n+k}
\end{array}\right] \quad \bar{B}_{i}^{*}=\left[\begin{array}{c}
\bar{B}_{i}^{f} \\
0
\end{array}\right] \\
& C_{i}^{*}=\left[\begin{array}{llllll}
C^{f} & D^{f} C_{i} & 0 & 0 & 0 & -D^{f} C_{i}^{r}
\end{array}\right]
\end{aligned}
$$

The Lyapunov function $V\left(x_{a}(t)\right)$ is defined by (9) with

$$
X_{i}=\left(\begin{array}{cccccc}
X_{i}^{11} & X_{i}^{12} & X^{13} & 0 & 0 & 0 \\
X_{i}^{12 T} & X_{i}^{22} & X^{23} & 0 & 0 & 0 \\
X^{13 T} & X^{23 T} & X^{33} & 0 & 0 & 0 \\
X_{i}^{41} & X_{i}^{42} & X_{i}^{43} & X_{i}^{44} & X_{i}^{45} & X_{i}^{46} \\
X_{i}^{51} & X_{i}^{52} & X_{i}^{53} & X_{i}^{54} & X_{i}^{55} & X_{i}^{56} \\
X_{i}^{61} & X_{i}^{62} & X_{i}^{63} & X_{i}^{64} & X_{i}^{65} & X_{i}^{66}
\end{array}\right)
$$

It can be shown that the matrices $X_{i}$ are positive definite with similar arguments to those given in the previous proof.

From (38), the function $V\left(x_{a}(t)\right)$ is positive definite. As seen previously, the $\mathcal{L}_{2}$-gain of the approximation error is bounded by $\gamma$ if $\mathcal{M}_{h h}<0$ holds with (30). Using the system matrices defined in (44) and the Lyapunov function defined by (9) and (45), $\mathcal{M}_{i j}$ in (30) becomes $\Theta_{i j}$ defined by (41) and the LMI conditions (14-16) of theorem 1 become (3840), following the same steps than in the proof of theorem 1 and using the variable changes $\bar{\gamma}=\gamma^{2}$ and

$$
\begin{aligned}
& {\left[\begin{array}{lll}
A_{1 i}^{r} & A_{2 i}^{r} & A_{3 i}^{r}
\end{array}\right]=A_{i}^{r}\left[\begin{array}{lll}
X^{13 T} & X^{23 T} & X^{33}
\end{array}\right]} \\
& {\left[\begin{array}{llll}
C_{1 i}^{r} & C_{2 i}^{r} & C_{3 i}^{r}
\end{array}\right]=C_{i}^{r}\left[\begin{array}{lll}
X^{13 T} & X^{23 T} & X^{33}
\end{array}\right]}
\end{aligned}
$$

\section{NUMERICAL EXAMPLE}

The results presented in Theorem 2 are applied in order to compute a second order nonlinear system approximation a fifth order nonlinear system with $r=3$ subsystems, $m=1$ output and $p=2$ inputs, defined by (1) with $D_{1}=\left[\begin{array}{ll}0.005 & 0.005\end{array}\right]$ and $D_{2}=D_{3}=\left[\begin{array}{lll}0.004 & 0.002\end{array}\right]$

$$
\begin{aligned}
& A_{1}=\left[\begin{array}{ccccc}
-12 & -32 & 38 & -38 & 0 \\
1.67 & -26 & -2.33 & -1.67 & 0 \\
-1.33 & -24 & -6.33 & 1.33 & 8 \\
-4.33 & -8 & 40.67 & -45.67 & 8 \\
1.67 & -25 & -2.33 & -1.67 & -1
\end{array}\right] \\
& B_{1}=\left[\begin{array}{cc}
0.57 & 0.41 \\
-0.11 & -0.037 \\
0.98 & 0.45 \\
0.92 & 0.44 \\
0.28 & 0.08
\end{array}\right] \quad C_{1}^{T}=\left[\begin{array}{c}
0.667 \\
0 \\
-1.33 \\
1.33 \\
0
\end{array}\right] \\
& A_{2}=\left[\begin{array}{ccccc}
-7.33 & -32 & 24.67 & -24.67 & 0 \\
6 & -41 & 2 & -6 & 0 \\
2.33 & -18 & -15.67 & -2.33 & 2 \\
6 & -2 & 20 & -38 & 2 \\
6 & -33 & 2 & -6 & -8
\end{array}\right] \\
& B_{2}=\left[\begin{array}{cc}
-0.5 & -0.26 \\
-0.22 & -0.13 \\
0.21 & 0.084 \\
0.25 & 0.11 \\
-0.22 & -0.15
\end{array}\right] \quad C_{2}^{T}=\left[\begin{array}{cc}
0.17 & \\
0 & \\
-0.33 & \\
0.33 & 0
\end{array}\right] \\
& A_{3}=\left[\begin{array}{ccccc}
-15.33 & -32 & 34.67 & -34.67 & 0 \\
0.333 & -23 & -7.67 & -0.333 & 0 \\
-3 & -24 & -8 & 3 & 8 \\
-4.33 & -8 & 40.67 & -45.67 & 8 \\
0.333 & -22 & -7.67 & -0.333 & -1
\end{array}\right] \\
& B_{3}=\left[\begin{array}{cc}
0.41 & 0.25 \\
-0.19 & -0.13 \\
0.62 & 0.42 \\
0.52 & 0.36 \\
0.088 & 0.09
\end{array}\right] \quad C_{3}=\left[\begin{array}{c}
0.33 \\
0 \\
-0.67 \\
0.67 \\
0
\end{array}\right]
\end{aligned}
$$

The input signals are depicted on Figure 1. The weighting functions, depending on the input signals, are defined by $w_{1}(t)=\left(\tanh \left(\left(u_{1}(t) u_{2}(t)\right) / 6\right)+1\right), w_{2}(t)=$ $\left(\tanh \left(\left(u_{1}(t)+u_{2}(t)\right) / 6\right)+1\right), w_{3}(t)=\left(\tanh \left(\left(u_{1}(t)-\right.\right.\right.$ $\left.\left.\left.u_{2}(t)\right) / 6\right)+1\right)$ and $h_{i}(t)=\left(w_{i}(t)\right) /\left(\sum_{k=1}^{r} w_{k}(t)\right)$. The weighting functions are depicted on figure 2 . The bound on the norm of the derivative of the weighting functions are $\Phi_{k}=0.3$. Without frequency weighting, the solution of the LMI problem given by (13-16) is a minimum $\mathcal{L}_{2}$-gain from $u(t)$ to $e(t)=y(t)-y_{r}(t)$ given by $\gamma=0.14$. The original 

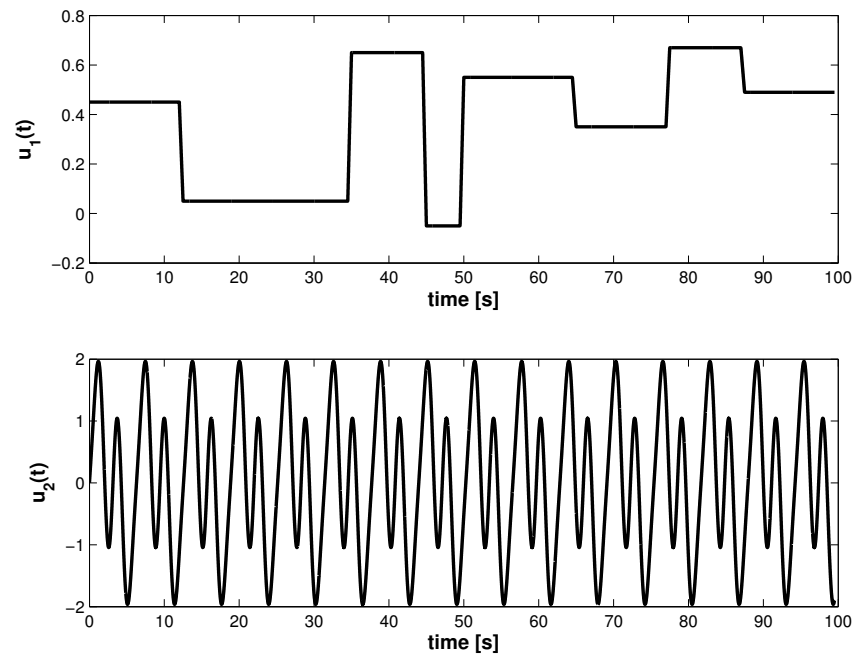

Fig. 1. System input $u(t)$
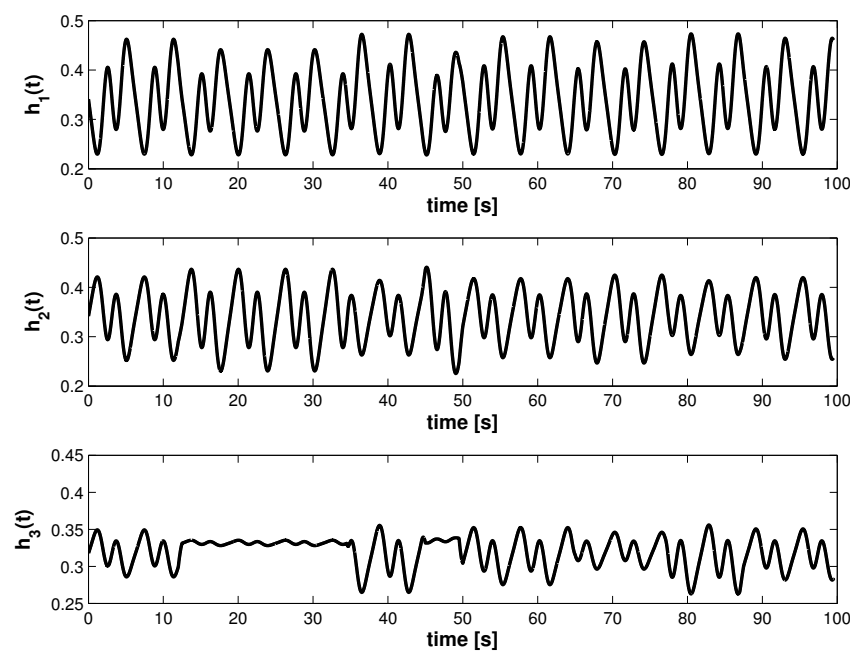

Fig. 2. Weighting functions

reduced system outputs are depicted on Figure 3. The obtained results can be improved with the use of a frequency weighting function in order to relax the $\mathcal{L}_{2}$-gain constraint on some frequency ranges. The frequency weighting function is chosen to be a bandpass filter with a passband from $10^{-2}$ to $10^{2} \mathrm{rad} / \mathrm{s}$, its transfer function is

$$
W^{f}(s)=\frac{0.0625(s+0.005)^{2}(s+2000)^{2}}{(s+0.02)^{2}(s+500)^{2}}
$$

Solving the LMI optimization problem given by (37-40), the minimum $\mathcal{L}_{2}$-gain from $u(t)$ to $e^{f}(t)$ is $\gamma=0.08$ and the

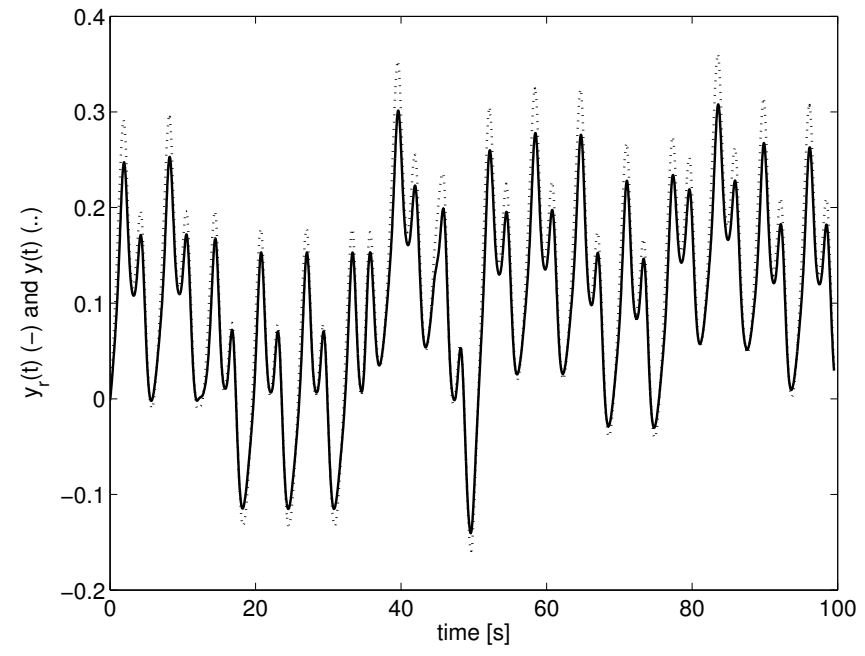

Fig. 3. $y$ (dashed line) and $y_{r}$ (solid line), obtained from Theorem 1

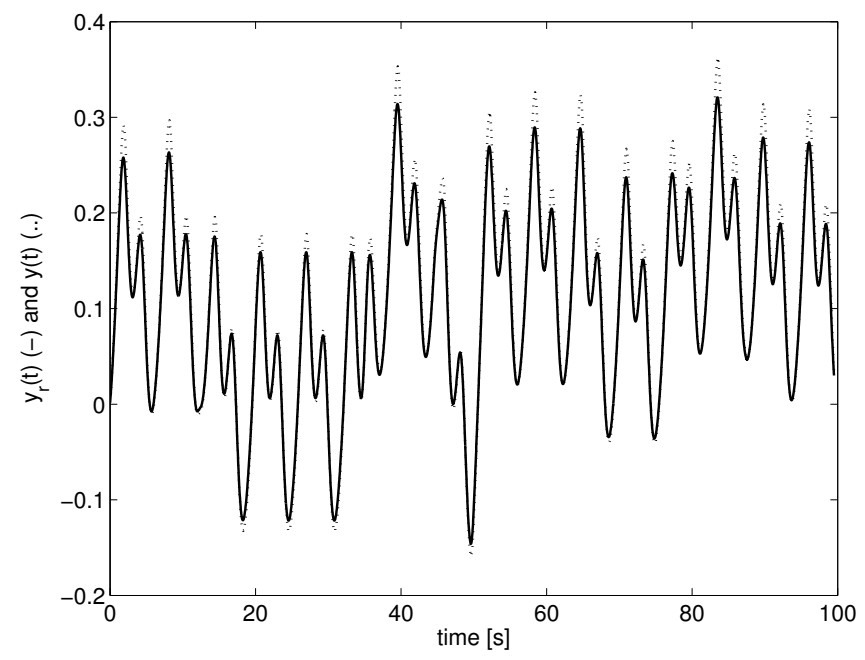

Fig. 4. $y$ (dashed line) and $y_{r}$ (solid line), obtained from Theorem 2

reduced order system is defined by (4) with

$$
\begin{array}{rlrl}
A_{1}^{r} & =\left[\begin{array}{ll}
-1.03 & -1.12 \\
-0.178 & -8.08
\end{array}\right] & C_{1}^{r T} & =\left[\begin{array}{l}
-0.57 \\
-0.31
\end{array}\right] \\
B_{1}^{r} & =\left[\begin{array}{ll}
-0.43 & -0.12 \\
0.044 & -0.32
\end{array}\right] & D_{1}^{r T}=\left[\begin{array}{l}
0.02 \\
0.02
\end{array}\right] \\
A_{2}^{r}=\left[\begin{array}{ll}
-17.84 & 17.47 \\
-17.39 & -17.57
\end{array}\right] & C_{2}^{r T}=\left[\begin{array}{c}
-0.31 \\
0.13
\end{array}\right] \\
B_{2}^{r}=\left[\begin{array}{ll}
0.19 & 0.047 \\
0.078 & 0.017
\end{array}\right] & D_{2}^{r T}=\left[\begin{array}{l}
0.03 \\
0.02
\end{array}\right] \\
A_{3}^{r}=\left[\begin{array}{cc}
-0.699 & -1.11 \\
1.11 & -5.15
\end{array}\right] & C_{3}^{r T}=\left[\begin{array}{l}
-0.47 \\
-0.35
\end{array}\right] \\
B_{3}^{r}=\left[\begin{array}{cc}
-0.31 & -0.21 \\
0.18 & 0.16
\end{array}\right] & D_{3}^{r T}=\left[\begin{array}{l}
-0.002 \\
-0.004
\end{array}\right]
\end{array}
$$

The system output $y(t)$ can be compared to the output of the reduced system $y_{r}(t)$, both depicted on Figure 4 . 


\section{CONCLUSION}

In this note, LMI conditions have been proposed to design a reduced order Takagi-Sugeno system that approximates a nonlinear system of higher order. The optimal reduced order system is found by minimizing the approximation error, quantified by the $\mathcal{L}_{2}$-gain from the input to the difference of the outputs of the original and reduced systems. Nonquadratic Lyapunov functions are used here, with the help of descriptor approach, in order to introduce slack variables to reduce the conservatism of the proposed results. Frequency weighting transfer functions are introduced in order to highlight a particular operating frequency range in which the original system should be precisely approximated. These weighting functions allow to prevent from imposing to hard constraints on the whole frequency range, indeed it may not be necessary to have an accurate approximation of the system outside of its useful bandwidth.

It should be mentioned that, due to the numbers of LMI conditions and scalar decision variables, the method exposed here is not suited for the model order reduction of high dimension systems. It should be rather used to decrease the state dimension of nonlinear systems to design observer / controller of reasonable order. Nevertheless, it should be mentioned that model order reduction and reduced order design of observer / controller are not trivial tasks.

Future works should be devoted to extend the presented results to discrete time nonlinear systems with some specific tools adapted to these systems.

\section{REFERENCES}

[1] S. Boyd, E. El Ghaoui, L. Feron, and V. Balakrishnan. Linear Matrix Inequalities in System and Control Theory. SIAM, Philadelphia, PA, 1994.

[2] Y. Ebihara and T. Hagiwara. On $H_{\infty}$ model reduction using LMIs. IEEE Transactions on Automatic Control, 49(7):1187-1191, July 2004.

[3] D.F. Enns. Model reduction with balanced realizations: An error bound and a frequency weighted generalization. In IEEE Conference on Decision and Control, pages 127-132, 1984.

[4] R. Freund. Applied and Computational Control, Signals, and Circuits: Volume 1, chapter Reduced-order modeling techniques based on Krylov subspaces and their use in circuit simulation, pages 435-498. Birkhauser, 1999.

[5] J.C. Geromel, R.G. Egas, and F.R.R. Kawaoka. $H_{\infty}$ model reduction with application to flexible systems. IEEE Transactions on Automatic Control, 50(3):402-406, 2005.

[6] K. Glover. All optimal Hankel-norm approximations of linear multivariable systems and their $L_{\infty}$ - error bounds. International Journal of Control, 39(6):1115-1193, 1984.

[7] K.M. Grigoriadis. Optimal $H_{\infty}$ model reduction via linear matrix inequalities: continuous-and discrete-time cases. Systems \& Control Letters, 26(5):321-333, 1995.

[8] B. Moore. Principal component analysis in linear systems: Controllability, observability, and model reduction. IEEE Transactions on Automatic Control, 26(1):17-32, 1981.

[9] M. Rewieński. A trajectory piecewise linear approach to model order reduction of nonlinear dynamical systems. $\mathrm{PhD}$ thesis, Massachusetts Institute of Technology, 2003.

[10] M. Rewieński and J. White. Model order reduction for nonlinear dynamical systems based on trajectory piecewise-linear approximations. Linear Algebra and its Applications, 415(2):426-454, 2006.

[11] H. Sandberg. An extension to balanced truncation with application to structured model reduction. IEEE Transactions on Automatic Control, 55(4):1038-1043, 2010.
[12] T. Tagaki and M. Sugeno. Fuzzy identification of systems and its application to modelling and control. IEEE Transactions on Systems, Man, and Cybernetics, 15(1):116-132, 1985.

[13] K. Tanaka, T. Hori, and H.O. Wang. A multiple Lyapunov function approach to stabilization of fuzzy control systems. IEEE Transactions on Fuzzy Systems, 11(4):582-589, 2003.

[14] K. Tanaka, H. Ohtake, and H.O. Wang. A descriptor system approach to fuzzy control system design via fuzzy Lyapunov functions. IEEE Transactions on Fuzzy Systems, 15(3):333-341, 2007.

[15] K. Tanaka and H.O. Wang. Fuzzy Control Systems Design and Analysis: a Linear Matrix Inequality Approach. Wiley-Interscience, 2001.

[16] H.D. Tuan, P. Apkarian, T. Narikiyo, and Y. Yamamoto. Parameterized linear matrix inequality techniques in fuzzy control system design. IEEE Transactions on Fuzzy Systems, 9(2):324-332, 2001.

[17] D. Vasilyev, M. Rewieński, and J. White. A TBR-based trajectory piecewise-linear algorithm for generating accurate low-order models for nonlinear analog circuits and MEMS. In Design Automation Conference, pages 490-495, 2003.

[18] L. Wu and W.X. Zheng. Weighted $H_{\infty}$ model reduction for linear switched systems with time-varying delay. Automatica, 45(1):186$193,2009$.

[19] S. Xu and J. Lam. $H_{\infty}$ model reduction for discrete-time singular systems. Systems \& Control Letters, 48(2):121-133, 2003.

[20] L. Zhang and P. Shi. $l_{2}-l_{\infty}$ model reduction for switched LPV systems with average dwell time. IEEE Transactions on Automatic Control, 53(10):2443-2448, 2008.

[21] Q.L. Zhang, V. Sreeram, G. Wang, and W.Q. Liu. $H_{\infty}$ suboptimal model reduction for singular systems. In IEEE American Control Conference, volume 2, pages 1168-1173, 2002.

[22] K. Zhou. Frequency-weighted $\mathcal{L}_{\infty}$ norm and optimal Hankel norm model reduction. IEEE Transactions on Automatic Control, 40(10):1687-1699, 1995.

[23] K. Zhou, J.C. Doyle, and K. Glover. Robust and Optimal Control. Prentice Hall Upper Saddle River, NJ, 1996.

[24] K. Zhou and P Khargonekar. Robust stabilization of linear systems with norm-bounded time-varying uncertainty. Systems \& Control Letters, 10(1):17 - 20, 1988. 